\title{
Determinación del Perfil Profesional de Estudiantes de Pregrado Aplicando la Técnica de Análisis Comparativo
}

\author{
Raúl J. Martelo(1)*, Iris Jiménez-Pitre(2) y Angie Quintana ${ }^{(1)}$ \\ (1) Universidad de Cartagena, Facultad de Ingeniería, Grupo de Investigación en Tecnologías de las \\ Comunicaciones e Informática, GIMATICA, Avenida del Consulado, Calle 30, No. 48-152, Cartagena \\ Colombia. (e-mail: rmartelog1@unicartagena.edu.co) \\ (2) Universidad de la Guajira, Facultad de Ciencias Básicas y Aplicadas, Grupo de Investigación BIEMARC, \\ $\mathrm{Km} 5$ vía a Maicao, Guajira-Colombia. \\ *autor a quien debe ser dirigida la correspondencia
}

Recibido Feb. 3, 2017; Aceptado Abr. 4, 2017; Versión final Jul. 17, 2017, Publicado Abr. 2018

\begin{abstract}
Resumen
En el presente estudio se determinó el perfil profesional para estudiantes de pregrado en Instituciones de Educación Superior a través de la técnica de Análisis Comparativo (benchmarking). En este sentido, se vincula la técnica mencionada con la metodología implementada en el programa de ingeniería de sistemas en modalidad presencial de la Universidad de Cartagena, en Colombia. Lo anterior define un modelo conformado por tres fases: planeación, recolección y análisis de información e integración y acción, para determinar perfiles profesionales. La investigación enriquece el aprendizaje de mejores prácticas aplicadas en diferentes programas académicos en Instituciones de Educación Superior. Entre éstas, conocer a fondo condiciones del mercado, posicionarse frente a la competencia, el análisis de fallas durante el proceso, y proponer soluciones para el desarrollo de habilidades.
\end{abstract}

Palabras clave: competencias; currículo; perfil ocupacional; análisis comparativo

\section{Determination of the Professional Profile of Undergraduate Students Applying the Benchmarking Technique}

\begin{abstract}
In the present study, the professional profile for undergraduate students in Higher Education Institutions was determined through the benchmarking technique. In this sense, the mentioned technique is linked with the methodology implemented in the Systems Engineering program in face-to-face modality of the University of Cartagena. This defines a model composed of three phases: planning, collection and analysis of information, and integration and action, to determine professional profiles. The research enriches the learning of best practices applied in different academic programs in Higher Education Institutions. Among these, knowing in depth the market conditions, positioning itself in front of the competition, analysis of failures during the process, and proposing solutions for the development of skills.
\end{abstract}

Keywords: skills; curriculum; occupational profile; phases; benchmarking 


\section{INTRODUCCIÓN}

Una práctica constante y habitual de las Instituciones de Educación Superior (IES) es el diseño de programas académicos. En este proceso, un concepto utilizado con frecuencia es el de perfil profesional, el cual describe y analiza las competencias que deben tener los egresados, además de conformar proyectos educativos que impulsen a lograr ese objetivo. Para este tipo de instituciones, disponer de la descripción del perfil profesional es importante para: promoción de estudiantes, diseño del currículo educativo y obtención de una visión acorde a la demanda del mercado laboral (Gonzáles et al., 2014). La importancia de definir un perfil profesional se evidencia en la insistencia del Ministerio de Educación Nacional Colombiano, al estipular e incorporar competencias laborales en el currículo educativo. Como resultado de lo anterior, los empresarios colombianos han experimentado cambios en los conocimientos adquiridos, debido a que existen vacíos y limitaciones preocupantes en egresados (Ministerio de Educación Nacional, 2014). Como hecho manifiesto de lo mencionado se tiene la encuesta de la Agència per a la Qualitat del Sistema Universitario de Catalunya (2014), la cual en sus resultados muestra que más del $70 \%$ de empresarios del Reino Unido prevén cambios a lo largo del año en las competencias requeridas para cubrir sus puestos de trabajo. De igual manera, expone que empleos de profesionales (ocupados por graduados en mayoría), requieren mayor persistencia de mejora en las competencias. Por lo anterior, las IES están en la obligación de reestructurar constantemente el perfil profesional de sus estudiantes, con el fin de adaptarlo a las necesidades en contextos productivos, académicos y científicos.

Para determinar competencias requeridas por estudiantes en el desempeño de una actividad, las IES necesitan herramientas que permitan recolectar información, analizar y plantear resultados para cada perfil profesional. Gonzáles y Patarrollo (2014) por ejemplo en la identificación de requerimientos y percepciones actuales respecto a competencias específicas del egresado de ingeniería industrial. Soler-Adillon et al., (2016) presentó una aproximación a características que definen el perfil profesional del diseñador de interacción en proyectos de comunicación y por último Boned et al., (2015), el cual profundiza en el conocimiento de características sociodemográficas de profesionales del sector del fitness en España.

Teniendo en cuenta que las técnicas anteriores cubren las necesidades mencionadas, es necesario evaluar, medir y comparar servicios, funciones y procesos de trabajo en IES, de manera que permitan establecer puntos de referencia para el mejoramiento en particular, por lo tanto, se enuncia la utilización de benchmarking. El concepto de benchmarking ha sido ampliamente utilizado para determinar qué y dónde se evidencian fallas, analizar cómo otros alcanzan niveles de alto rendimiento y utilizar esta información para implementar mejoras (Edwards et al., 2014). Su proceso implica la identificación de organizaciones destacadas, la comparación de métricas o indicadores específicos de desempeño y el aprendizaje de los pares (Dai y Kuosmanen, 2014). La clave para obtener un buen proceso, es entender porque será beneficioso para la organización que lo utiliza. Plantearse ¿Cómo la mejor práctica empresarial agrega valor a la compañía?, en este sentido, identificar los principios operativos subyacentes que están siendo apalancados por la mejor práctica empresarial (Lapide, 2005).

Según Ammons y Roenigk (2015) existen 3 tipos de benchmarking: Comparación de estadísticas de rendimiento, iniciativa de visión y benchmarking de las mejores prácticas. Los tres tipos ofrecen un potencial para influir en el aprendizaje interorganizacional y mejorar el rendimiento. La comparación de estadísticas de rendimiento, también denominada benchmarking de métricas y benchmarking de resultados, tiene como propósito el diagnóstico, es decir, revelar brechas que separen los desempeños de la organización de las normas y logros de las organizaciones que se analizan. La importancia de este tipo, es su utilización como un medio para medir fortalezas y debilidades relativas, pero el beneficio subyacente y quizás el más importante, es el intercambio de información sobre prácticas y estrategias efectivas. De acuerdo al mismo autor, la iniciativa de visión se enfoca en metas generales y adopta un punto de referencia como etiqueta para sus objetivos. A diferencia de la comparación de estadísticas de rendimiento, donde los objetivos están anclados en los resultados de desempeño obtenido por otros, en éste están a menudo vinculados a las condiciones sociales deseadas. El tercer tipo de benchmarking y enfoque principal de este artículo, se centra estrechamente en un proceso seleccionado, identifica entre otras organizaciones a los mejores actores de este proceso y adopta o adapta los métodos de esos líderes para usarlos en la propia organización; siguen una serie de pasos prescritos que incluyen procedimientos rigurosos para identificar a los mejores de su clase, analizando procesos y aislando elementos que contribuyen a excelentes resultados.

Existen aplicaciones de Benchmarking en diversas áreas del conocimiento. Assaf y Pestana (2013), la utilizaron para realizar un análisis de eficiencia de compañías hoteleras líderes en el mundo. MacLean et al., (2016), propusieron desarrollar y probar medidas de desempeño de seguridad en tráfico aéreo. De igual manera Del Giorgio (2012a) estudió la importancia, alcances y dimensiones del benchmarking de diseño de producto o industrial. En otro trabajo, de Del Giorgio (2012b), evaluó la importancia que ha cobrado la técnica en el sector público. Martínez (2016) expuso las particularidades de la referenciación competitiva o 
benchmarking en general. Assaf y Dwyer (2013) utilizaron la técnica para desarrollar una metodología que proporcione a gobiernos y oficinas de turismo una evaluación precisa de su desempeño turístico internacional.

Además, específicamente en el área de la educación existen los siguientes ejemplares: Kuzmic (2015) analizó el concepto de organización de aprendizaje en IES e indicó cómo la técnica puede ayudar a alcanzar ese fin. Santos (2015) revisó Benchmarking como instrumento para mejorar el desempeño, en la calidad de los servicios ofrecidos por instituciones de educación media superior a distancia. Paliulis y Labanauskis (2015) la utilizaron como instrumento para el mejoramiento de la gestión de la calidad en educación superior y Gómez (2013), realizó una revisión teórica y procedimental de aplicaciones de la metodología DEA al sector educativo. Finalmente, Córdoba (2014) propuso para el mejoramiento de la gestión educativa, el desarrollo de marcas en instituciones de básica y media.

Teniendo en cuenta lo anterior, surge el siguiente cuestionamiento: ¿Puede la técnica de planificación estratégica Benchmarking, implementar cambios ligados a estándares de calidad y mejores prácticas a un perfil profesional de pregrado, con el fin de desarrollar y fortalecer competencias profesionales y estudiantiles? Para dar respuesta a lo anterior, el objetivo de la investigación define el perfil profesional de estudiantes de pregrado mediante la utilización de esta técnica. Para tal fin, se empleó la metodología aplicada en el programa ingeniería de sistemas de la Universidad de Cartagena, con el propósito de validar el uso del enfoque propuesto, valiéndose de las ventajas que ofrece la técnica.

\section{MATERIALES Y METODOS}

El diseño de la investigación que se abordó es de tipo no experimental transeccional descriptivo. No experimental porque las variables no fueron manipuladas y el investigador se limitó a observar los acontecimientos sin intervenir en los mismos, transeccional porque los datos se obtuvieron en un tiempo definido y descriptivo porque se buscó medir las competencias que deben desarrollar los estudiantes en un campo especifico. A partir de eso, se definió el perfil profesional de dicho campo con la aplicación de la técnica benchmarking (Hernández et al., 2006).

\section{Población y muestra}

Para el estudio se estableció una población conformada por estudiantes, docentes, egresados y empresarios del programa ingeniería de sistemas de la Universidad de Cartagena. Para determinar la muestra de la población se consideró el muestreo aleatorio simple, por lo que se utilizó la siguiente fórmula (Álvarez y Tello, 2015):

$$
n=\frac{N p q z^{2}}{(N-1) e^{2}+p q z^{2}}
$$

\section{Dónde:}

$\mathrm{n}=$ Tamaño de la muestra

$z=$ nivel de confianza aplicado al estudio (1.96, equivalente al 95\% del nivel de confianza)

$p=$ Probabilidad de ocurrencia del evento previsto $(50 \%)$

$\mathrm{q}=$ Probabilidad de no ocurrencia del evento previsto $(50 \%)$

$\mathrm{e}=$ Error de tolerancia máxima permitida $(9 \%)$

$\mathrm{N}=$ Tamaño de la población

\section{Materiales de recolección de datos}

La técnica de recolección de datos utilizada fue la encuesta, ya que a diferencia de otras, posibilita: la utilización en una variedad de ámbitos de forma económica, una amplitud de interrogantes en un mismo estudio y facilita la comparación de resultados (Anguita, Labrador y Campos, 2003a). Esta fue medida a través de los instrumentos de recolección de datos Cuestionario y Entrevista. Donde la primera, a partir de un conjunto de ítems planteados de forma interrogativa acordes al tema en estudio (Jiménez y González, 2015); tuvo como objetivo, asistir la definición de los factores que impiden el completo desarrollo de las competencias de los estudiantes. Esta herramienta fue aplicada a la muestra representativa de estudiantes y egresados a través de la herramienta SoftProsp, debido a que los cuestionarios autoadministrados por un medio electrónico, evitan el sesgo proporcionado por la presencia de un entrevistador, disminuyen los costos, permiten acceder a todo tipo de personas por muy alejadas que estén y le facilitan a los encuestados responder cuando lo encuentre oportuno. 
En cuanto a la técnica entrevista, tuvo como propósitos: obtener información en relación con un tema determinado, buscar que la información recabada fuera lo más precisa posible y aclarar los significados atribuidos por los informantes (Díaz-Bravo et al., 2013). Esta fue aplicada en reuniones organizadas a representantes del programa de Ingeniería de Sistemas de las instituciones estudiadas; y con apoyo de libros, artículos, guías u otros recursos bibliográficos, definir las competencias necesarias para el buen desempeño de estudiantes en un campo específico. Esta herramienta, al tener que ser contestada en el acto, evitó consultas o influencias de terceras personas. Además, al exigir menos esfuerzo por parte del entrevistado, el proceso pudo ser más extenso, lo que permitió conseguir mayor porcentaje de respuesta por pregunta (Anguita, Labrador y Campos, 2003b).

\section{Análisis de los datos}

Al emplear la herramienta web para acompañamiento de estudios prospectivos SoftProsp, se efectuaron los análisis, apoyados en el diagrama de información propia (información recopilada de la organización que requiere Benchmarking), mediante la comparación cuantitativa y jerarquizada, lo cual produjo como resultado los motivos a tener en cuenta para tomar decisiones.

\section{Modelo conceptual planteado}

Para el desarrollo del presente estudio, se tuvo como soporte la metodología implementada en la elaboración del perfil profesional en el programa de ingeniería de sistemas de la Universidad de Cartagena. En la Figura 1 se muestran las fases que conlleva dicha metodología, en la cual, siguiendo el orden respectivo de la imagen, se realiza un análisis local y regional de las necesidades existentes a nivel económico, social, empresarial e industrial. Luego se evalúan currículos de diferentes programas de ingeniería de sistemas a nivel nacional y se estudian guías con las que cuenta IEEE y ACM, para definir perfiles profesionales en esta área y finalmente se propone para el programa. Al implementar la técnica benchmarking al proceso descrito, se propone el modelo de la Figura 2.

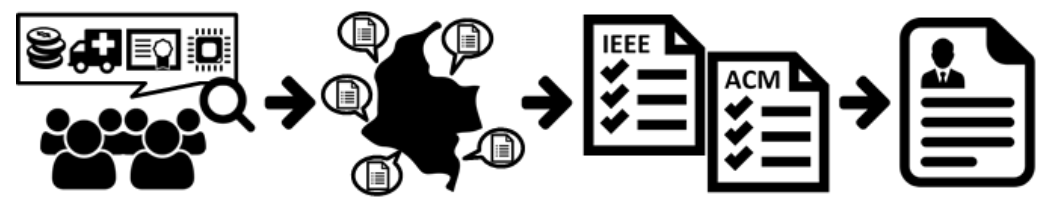

Fig.1: Metodología para la elaboración de perfil profesional del programa ingeniería de sistemas.

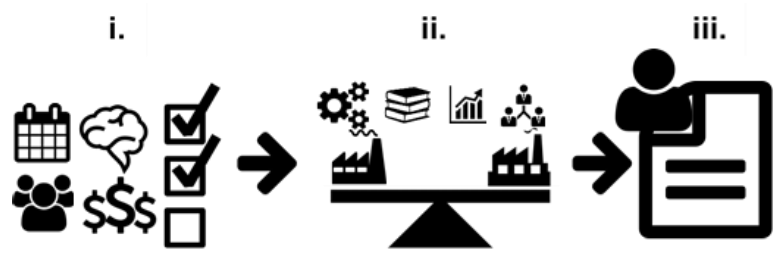

Fig. 2: Modelo propuesto para la determinación de perfiles profesionales con la herramienta benchmarking

Para el acompañamiento en la determinación del perfil profesional, el modelo propuesto dispone de las siguientes fases: (i) Planeación, (ii) Recolección y análisis de información; e (iii) Integración y acción:

(i) Planeación: Se determinan los integrantes que conforman el equipo de expertos (estudiantes, egresados, docentes y empresarios), estos son los encargados de conducir el proceso para evaluar el impacto del programa académico, suministrar información y aportar conocimiento a partir del papel que desempeñan en el área de estudio; a continuación, se deciden aquellos factores que no permiten el completo desarrollo de las competencias de los estudiantes, a fin de aplicar cuestionarios y/o lluvia de ideas al grupo de expertos. A partir de los resultados, se utiliza el diagrama Causa-Efecto para poner en relieve las razones del problema y diagrama de Pareto para definir los puntos de medida, aquellos que aportarán una visión precisa del rendimiento del currículo académico. Finalmente, se debe hacer una búsqueda y selección de IES con excelentes resultados en su proceso educativo, aquellas donde sus egresados pertenecientes al campo en estudio, sean demandados en el sector industrial y empresarial.

(ii) Recolección y análisis de información: Esta fase es constituida por dos etapas, recogida de datos internos y recogida de datos externos. Ambas esenciales para conocer la situación del programa que requiere el estudio y la de la institución objetivo, y trasladar soluciones de la institución objetivo a la propia. Para la primera etapa, como fuente de información principal, se le suministra al equipo de expertos, información del rendimiento de los estudiantes y egresados, recogida y analizada periódicamente. Para la última, se recurre 
a información de carácter público, como artículos, guías u otros que especifiquen las competencias necesarias en el buen desempeño de estudiantes en un campo específico. Además, se incluye información privada, como: currículos educativos que deben ser suministrados por IES y se organizan reuniones o entrevistas a los actores de las instituciones, con el fin de obtener información relevante. Por último, se realiza un informe detallado de forma estructurada, donde se compare la organización propia y la objetivo.

(iii) Integración y acción: A partir de los resultados obtenidos en la fase anterior, el problema deja de ser genérico, las deficiencias en los egresados, las causas de ello y algunas posibles soluciones ya son conocidas. Por lo tanto, en reuniones con el personal que dirige y orienta la formación de los estudiantes, se determinan las competencias que deben estar incluidas en el desarrollo del micro currículo de la asignatura, y finalmente, teniendo en cuenta las competencias establecidas y las necesidades del sector empresarial e industrial, se define el perfil profesional del futuro egresado.

\section{RESULTADOS Y DISCUSIÓN}

Como prueba del modelo propuesto con benchmarking, a lo largo del primer periodo académico y principios del segundo (de febrero a septiembre) del año 2016, se realizó la definición del perfil profesional del Ingeniero de Sistemas formado en la Universidad de Cartagena modalidad presencial. Para lo anterior, se creó una sesión de estudio con la herramienta benchmarking de la plataforma SoftProsp, en donde se ingresan datos como: el nombre del caso a estudiar, fecha de inicio y fin, expertos, datos de la organización y del moderador. De acuerdo al modelo propuesto en la Figura 2, las fases se desarrollaron de la siguiente forma:

\section{(i) Planeación}

Se determinaron los integrantes que conformaron el equipo de expertos, aquellos especialistas en un área específica o que desde su experiencia proporcionan información al programa de ingeniería de sistemas. Para tal fin se identificaron los roles del grupo de expertos (Ver Tabla 1), se tomó una muestra representativa de cada uno a través de una fórmula estadística (Ver Tabla 2) y se contactaron a los expertos.

Tabla 1: Roles identificados del grupo de expertos

\begin{tabular}{|c|c|}
\hline Expertos & Descripción \\
\hline Estudiantes & Estudiantes matriculados en el programa ingeniería de sistemas \\
\hline Docentes & Docentes de las áreas del programa \\
\hline Egresados & Profesionales formados en el programa de ingeniería de sistemas \\
\hline Empresarios & Directivos vinculados a empresas del sector público y privado en el área profesional \\
\hline
\end{tabular}

Tabla 2: Población y muestra del grupo de expertos

\begin{tabular}{|c|c|c|}
\hline Expertos & Población & Muestra \\
\hline Estudiantes & 341 & 88 \\
\hline Docentes & 40 & 30 \\
\hline Egresados & 157 & 68 \\
\hline Empresarios & 24 & 20 \\
\hline Total & 562 & 206 \\
\hline
\end{tabular}

Identificación de los factores claves

Para identificar los factores que no permiten el completo desarrollo de las competencias de los ingenieros de sistemas, fueron utilizadas las técnicas encuesta, lluvia de ideas y diagrama de causa-efecto. La primera fue validada a través de la técnica juicio de expertos, en la que se reunieron un pequeño grupo de conocedores sobre la temática y plantearon algunas recomendaciones y observaciones a fin de garantizar la viabilidad del contenido del instrumento de recolección de datos. Esta fue aplicada especialmente a la muestra representativa de estudiantes y egresados, debido a la magnitud de sus integrantes.

La técnica lluvia de ideas fue abordada para potenciar la participación y creatividad del grupo de expertos, mediante un proceso estructurado en el que se consiguió la máxima libertad y aportación de los participantes. Se asignó un moderador con conocimiento en el manejo de la técnica, el cual fue el encargado de dirigir con éxito el estudio, en el que transmitió las reglas conceptuales y prácticas de la lluvia de ideas, agrupó, clasificó y concluyó las ideas propuestas en cada ronda. En esta, se tuvieron en cuenta los resultados ya organizados 
y analizados de la encuesta. Por último, la técnica diagrama de causa-efecto, también denominada como diagrama Ishikawa fue utilizada para poner en relieve las causas últimas o factores claves, a partir de las relaciones de causalidad de las ideas concluidas en la técnica anterior. En la figura 3 se puede visualizar el diagrama generado por la plataforma SoftProsp.

\section{Visión del rendimiento del currículo académico}

A partir de las causas establecidas (ver Figura 3) y la frecuencia con que los expertos coincidieron en estas. El sistema generó una Tabla de Pareto (Tabla 3) con los resultados en orden decreciente, donde se evidencian las causas con su respectiva frecuencia: acumulada (suma de frecuencias de causas precedentes), porcentual (porcentaje en que la causa contribuye al problema) y porcentual acumulada (suma de porcentajes de causa precedentes).
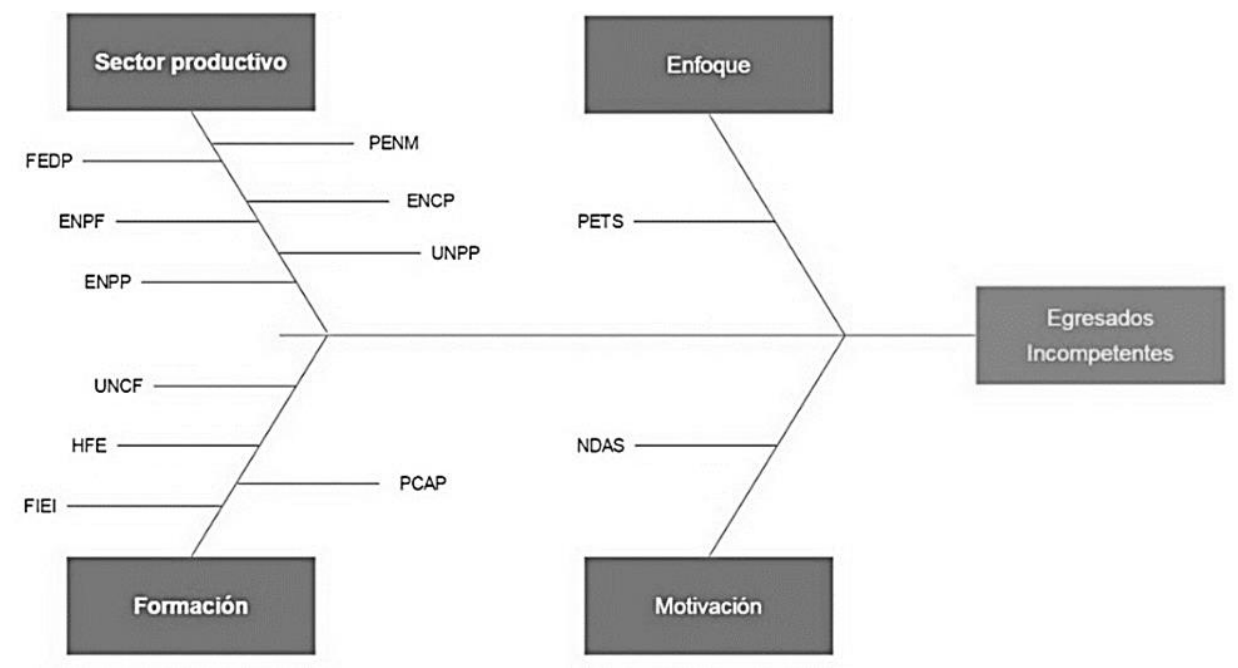

Fig. 3: Diagrama de causa-efecto o Ishikawa

Tabla 3: Tabla de Pareto

\begin{tabular}{|c|c|c|c|c|c|}
\hline $\begin{array}{c}\text { Nombre } \\
\text { Corto }\end{array}$ & Causa & Frecuencia & $\begin{array}{l}\text { Frecuencia } \\
\text { acumulada }\end{array}$ & $\begin{array}{c}\% \text { Frecuencia } \\
\text { porcentual }\end{array}$ & $\begin{array}{c}\% \\
\text { acumulados }\end{array}$ \\
\hline PENM & $\begin{array}{l}\text { El plan de estudio se ajusta a las } \\
\text { necesidades productivas }\end{array}$ & 202 & 202 & 9,8 & 9,8 \\
\hline PETS & $\begin{array}{l}\text { Los profesionales están más enfocados } \\
\text { a la tecnología que a buscar soluciones }\end{array}$ & 197 & 199 & 9,6 & 19,4 \\
\hline UNCF & $\begin{array}{l}\text { Las universidades no tiene claridad } \\
\text { sobre la formación del ingeniero de } \\
\text { sistemas }\end{array}$ & 194 & 593 & 9,4 & 28,8 \\
\hline HFE & Hace falta especialización & 191 & 784 & 9,3 & 38,1 \\
\hline FIEI & $\begin{array}{l}\text { Hace falta inculcar en los estudiantes la } \\
\text { investigación }\end{array}$ & 178 & 962 & 8,6 & 46,7 \\
\hline FDEP & $\begin{array}{l}\text { Faltan docentes con experiencias en el } \\
\text { sector productivo }\end{array}$ & 173 & 1135 & 8,4 & 55,1 \\
\hline NDAS & $\begin{array}{l}\text { No se ha despertado la atención sobre } \\
\text { la ingeniería de sistemas, el perfil del } \\
\text { ingeniero de sistemas y el rol que debe } \\
\text { desempeñar }\end{array}$ & 173 & 1308 & 8,4 & 63,5 \\
\hline ENCP & $\begin{array}{l}\text { Las empresas no tienen en cuenta a los } \\
\text { profesionales con sus doctorados y } \\
\text { maestrías }\end{array}$ & 162 & 1470 & 7,9 & 71,4 \\
\hline PCAP & $\begin{array}{l}\text { Algunos profesionales no tiene la } \\
\text { capacidad de autoaprendizaje }\end{array}$ & 150 & 1620 & 7,3 & 78,7 \\
\hline UNPP & $\begin{array}{l}\text { Las universidades no cuentan con un } \\
\text { plan de estudio no actualizado }\end{array}$ & 149 & 1769 & 7,2 & 85,9 \\
\hline ENPF & $\begin{array}{l}\text { Las empresas no participan en el } \\
\text { proceso de formación de los } \\
\text { profesionales }\end{array}$ & 148 & 1917 & 7,2 & 93,1 \\
\hline ENPP & $\begin{array}{l}\text { Las empresas no aseguran las prácticas } \\
\text { de los profesionales frente a sus } \\
\text { necesidades }\end{array}$ & 142 & 2059 & 6,9 & 100 \\
\hline-- & Total & 2059 & 2059 & 100 & 100 \\
\hline
\end{tabular}


Finalmente, SoftProsp muestra un Diagrama de Pareto (Figura 4), en el cual a partir de los resultados de la tabla anterior, determina los motivos principales que impiden el buen desarrollo de competencias en estudiantes de Ingeniería de Sistemas; toma elementos considerados "poco vitales", es decir, aquellos que se encuentren por debajo de $80 \%$ según el eje vertical derecho (porcentaje acumulado). Dicho diagrama está compuesto por un eje vertical izquierdo el cual representa las frecuencias, un eje horizontal que contiene los distintos elementos o factores que contribuyen al problema (representados en un nombre corto) y un eje vertical derecho que representa la magnitud de los porcentajes acumulados del efecto estudiado.

Los resultados obtenidos fueron los siguientes: el plan de estudio no se ajusta a las necesidades productivas, los profesionales están más enfocados a la tecnología que a buscar soluciones, las universidades no tienen claridad sobre la formación del ingeniero de sistemas. Además, hace falta especialización, inculcar en los estudiantes la investigación, docentes con experiencias en el sector productivo y las empresas no tienen en cuenta a los profesionales con sus doctorados y maestrías. Cabe resaltar que algunos profesionales no tienen la capacidad de autoaprendizaje y no se ha dado importancia al perfil y rol que debe desempeñar el ingeniero de sistemas.

\section{(ii) Recolección y análisis de información}

Para la obtención de datos interno, el programa ingeniería de sistemas le suministró al equipo de expertos información sobre el rendimiento de estudiantes y egresados, recopilada y analizada semestralmente, donde además se presentan algunas causas identificadas de deserción y soluciones planteadas por el comité directivo del programa académico y un profesional de trabajo social otorgado por el área de bienestar universitario de la Universidad de Cartagena.

En cuanto a los datos externos, se hizo una búsqueda y selección de IES con excelentes resultados en su proceso educativo en el campo de ingeniería de sistemas. Las IES elegidas, para posteriormente efectuar una evaluación comparativa de sus currículos académicos, fueron: Universidad del Magdalena, Universidad del Norte, Corporación Universitaria Rafael Núñez, Fundación Universitaria Tecnológico Comfenalco, Universidad Tecnológica de Bolívar y la Universidad de San Buenaventura. Luego, se diligenciaron las solicitudes para la obtención de sus currículos, se organizaron entrevistas a los actores de estas instituciones y se adquirieron referentes internacionales como el grupo de Educación en Computación de la ACM/IEEE y ABET.

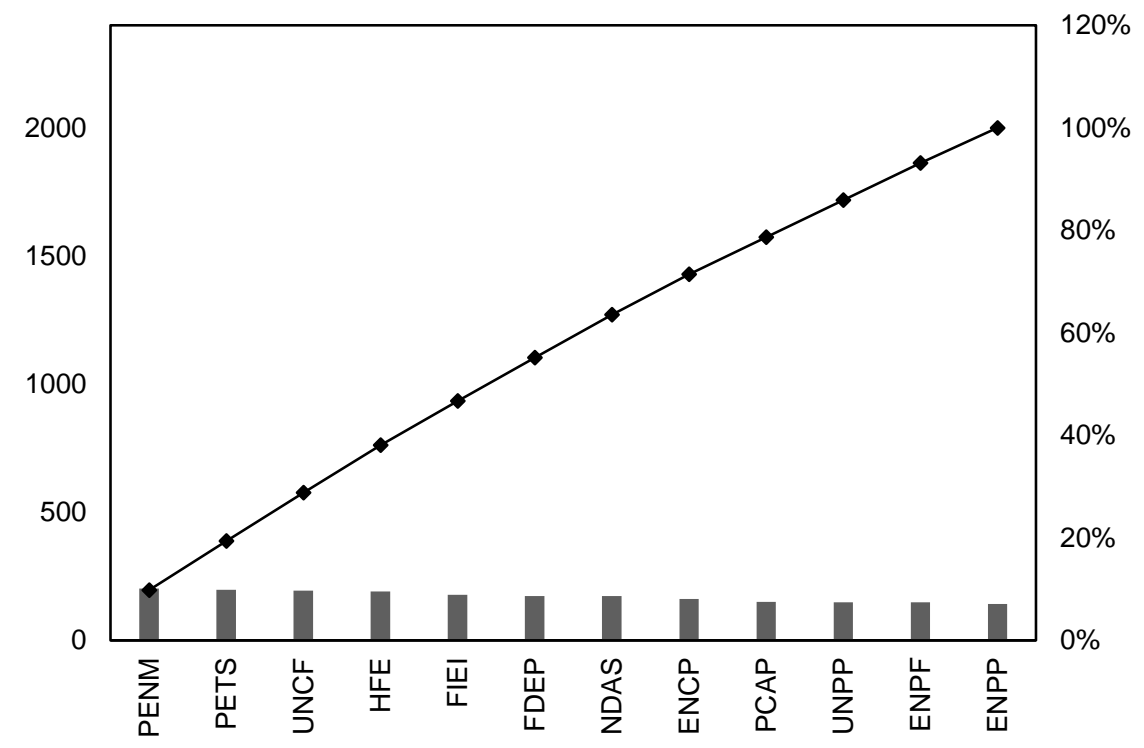

Fig. 4: Diagrama de Pareto

Como resultado de lo anterior, se realizó un documento donde se detalló de forma estructurada la información obtenida. En este, son comparados el programa Ingeniería de Sistemas de la Universidad de Cartagena y de las instituciones descritas anteriormente.

(iii) Integración y acción

A partir de los resultados obtenidos en la fase anterior, en reuniones con el personal que dirigió y orientó la formación de los estudiantes, se determinaron las competencias cognitivas, socio afectivas y comunicativas del ejercicio y de la cultura de la ingeniería de sistemas. (Ver Tabla 4) 
Tabla 4: Clasificación de competencias genéricas y específicas de acuerdo a los factores claves resultantes

\begin{tabular}{|c|c|c|c|c|c|c|c|c|}
\hline Factores claves & PENM & PETS & UNCF & HFE & FIEI & FDEP & PCAP & NDAS \\
\hline \multicolumn{9}{|l|}{ Competencias genéricas } \\
\hline Capacidad de análisis y síntesis & & - & & & & & & - \\
\hline Capacidad de organizar y planificar & & & & & & & & - \\
\hline Habilidades básicas en informática & & & - & & & & & - \\
\hline Resolución de problemas & - & - & & & & & & \\
\hline Capacidad de crítica y autocrítica & & & & & & - & & \\
\hline Capacidad de trabajar en equipo & - & & & & & & & \\
\hline Capacidad para aplicar la teoría a la práctica & - & & & & & & - & \\
\hline $\begin{array}{l}\text { Capacidad para un compromiso con la calidad } \\
\text { ambiental }\end{array}$ & - & & $\mathbf{0}$ & & & & & - \\
\hline Capacidad de aprender & & & & - & & & - & \\
\hline Habilidades de investigación & & & $\mathbf{0}$ & - & - & & & - \\
\hline Capacidad de generar nuevas ideas & & - & & & & & & \\
\hline Habilidad para trabajar de forma autónoma & & & - & & & & - & \\
\hline Habilidad para comunicarse efectivamente & - & & $\mathbf{0}$ & & & & & \\
\hline $\begin{array}{l}\text { Motivación y habilidad para afrontar el aprendizaje } \\
\text { durante toda la vida }\end{array}$ & - & & & - & & & & \\
\hline \multicolumn{9}{|l|}{ Competencias específicas } \\
\hline $\begin{array}{l}\text { Aplicar conocimiento en ciencias, matemáticas e } \\
\text { ingeniería }\end{array}$ & & & & - & & & & - \\
\hline $\begin{array}{l}\text { Facilitar trabajo en equipos multidisciplinarios, gestión } \\
\text { de proyectos, emprendimiento, diseño, administración, } \\
\text { planeación y desarrollo de software }\end{array}$ & - & & - & & & & & - \\
\hline $\begin{array}{l}\text { Habilidad para usar técnicas, competencias y } \\
\text { herramientas necesarias para la práctica de ingeniería } \\
\text { de sistemas }\end{array}$ & - & - & & & & & - & - \\
\hline $\begin{array}{l}\text { Habilidad para aplicar conocimientos de bases de } \\
\text { datos, redes y telecomunicaciones }\end{array}$ & & - & - & & & & & - \\
\hline Destreza en procesos de auditoría de sistemas & & & - & & & & & - \\
\hline $\begin{array}{l}\text { Desempeñar actividades de asesoramiento técnico en } \\
\text { ventas }\end{array}$ & & & - & & & & & - \\
\hline $\begin{array}{l}\text { Idoneidad para investigación y desarrollo de procesos } \\
\text { para implementación de soluciones informáticas en } \\
\text { organizaciones }\end{array}$ & & - & - & - & - & & & - \\
\hline
\end{tabular}

Lo anterior constituye las capacidades que debe tener un profesional ante diversas situaciones, aunque existen autores que se enfocan en determinar características basadas en el mercado. Como es el caso de Morato et al., (2016), donde definen tendencias en el perfil tecnológico del profesional de información en España. Teniendo en cuenta el análisis de cambios generados por la tecnología sobre las competencias y utilizándolas para analizar ofertas de trabajo e identificar conocimientos que favorezcan la inserción laboral. El anterior enfoque ignora la influencia de entornos académicos e investigativos, que de igual manera intervienen en las competencias profesionales.

Teniendo en cuenta las anteriores competencias, además de las necesidades del sector empresarial e industrial, se definió el perfil profesional de ingeniería de sistemas de la Universidad de Cartagena. (Tabla 5). Estas competencias resultan idóneas para sobresalir en el campo laboral y definen las características que debe poseer el profesional desde cualquier perspectiva, caso contrario se presenta en Pérez (2010), donde se busca determinar el perfil del profesional universitario futuro gerente Pymes, por medio de encuestas dirigidas a gerentes de Pymes representativas, por lo tanto enfatizan en un enfoque empresarial y omiten perspectivas académica e investigativa del perfil. 
Tabla 5: Clasificación de perfiles profesionales y ocupacionales de acuerdo a los factores claves resultantes

\begin{tabular}{|c|c|c|c|c|c|c|c|c|}
\hline Resultados & & & & & & & & \\
\hline \multicolumn{9}{|l|}{ Perfil profesional } \\
\hline $\begin{array}{l}\text { Actuar éticamente con alto sentido de responsabilidad profesional y } \\
\text { social, haciendo uso racional, eficiente y sostenible de los recursos } \\
\text { puestos a su disposición. }\end{array}$ & & - & & & & & & \\
\hline \multicolumn{9}{|l|}{$\begin{array}{l}\text { Comprometerse con el desarrollo tecnológico, científico, social y } \\
\text { cultural de la región Caribe y del país. }\end{array}$} \\
\hline $\begin{array}{l}\text { Perfil investigativo, empresarial y de soporte (Desarrollo de } \\
\text { software), orientado hacia: innovaciones en ciencia y tecnología, } \\
\text { manteniendo siempre una actitud ecológica y en defensa del medio } \\
\text { ambiente. }\end{array}$ & 口 & & D & & 口 & & & व \\
\hline $\begin{array}{l}\text { Liderar proyectos de investigación que promuevan el desarrollo de } \\
\text { conocimientos propios de la disciplina. }\end{array}$ & & 口 & & & 口 & & - & \\
\hline $\begin{array}{l}\text { Desarrollar actividades académicas en áreas de la ingeniería de } \\
\text { sistemas y profesiones afines. }\end{array}$ & & & & - & & & & \\
\hline $\begin{array}{l}\text { Desarrollar trabajos interdisciplinarios que conlleven a la búsqueda } \\
\text { de soluciones integrales en los campos de acción del conocimiento. }\end{array}$ & - & - & & - & - & & & \\
\hline \multicolumn{9}{|l|}{ Perfil ocupacional } \\
\hline $\begin{array}{l}\text { Director y/o asesor de proyectos de investigación propios de su } \\
\text { profesión con carácter interdisciplinario. }\end{array}$ & - & & - & & 口 & & - & व \\
\hline $\begin{array}{l}\text { Administrador de los recursos de software y hardware, que } \\
\text { contribuyan a la optimización del manejo de la información } \\
\text { empresarial. }\end{array}$ & 口 & 口 & 口 & - & & & & व \\
\hline $\begin{array}{l}\text { Coordinador de grupos de trabajos, orientados hacia la generación } \\
\text { de proyectos informáticos y sistemas de información. }\end{array}$ & 口 & - & - & - & & & & 口 \\
\hline $\begin{array}{l}\text { Diseñador de redes de comunicación que faciliten el flujo de la } \\
\text { información institucional, para apoyar procesos de planeación, } \\
\text { control y toma de decisiones. }\end{array}$ & 口 & - & 口 & - & & & & 口 \\
\hline $\begin{array}{l}\text { Gerente o jefe de tecnología y gestor de empresas de outsourcing } \\
\text { especializadas en el desarrollo de ingeniería de software, } \\
\text { telecomunicaciones y tecnologías de información general. }\end{array}$ & 口 & & - & - & & & & D \\
\hline
\end{tabular}

Respecto al perfil ocupacional Martelo et al., (2016), presenta un caso similar donde proponen una guía metodológica que establece pasos a seguir en el diseño de perfiles profesionales en IES, por medio de la técnica Ábaco de Régnier. La anterior técnica, permite un consenso anónimo y ubicuo de actores en los núcleos académico, investigativo y empresarial, lo cual integra cada margen del perfil profesional a definir, sin embargo, no comparan esta actividad con otras instituciones que implementan distintas metodologías, lo cual no permite un análisis completo del perfil presente.

Asimismo, estudios relacionados en el área de la educación realizados con benchmarking, como es el caso de Leppisaari et al., (2009), donde examinaron la creación de un modelo de benchmarking virtual, como herramienta para el desarrollo profesional de docentes; se convirtió en un entorno de aprendizaje donde los elementos estructurales, fomentaron el aprendizaje entre iguales y la construcción colaborativa del conocimiento, componente tomado como punto a favor en la obtención de perfiles profesionales.

\section{CONCLUSIONES}

De acuerdo al objetivo de la investigación, el cual estuvo orientado a definir el perfil profesional de estudiantes de pregrado mediante la utilización de la técnica Benchmarking, se concluye: i) El modelo propuesto se planteó de forma genérica, de modo que pueda ser adaptable a cualquier programa académico; ii) El modelo suministra soluciones innovadoras, debido al estudio de lineamientos estandarizados y metodologías empleadas por la competencia; iii) Permite la mejora continua del perfil profesional y su operacionalización por el programa académico y iv) Permite la participación del personal afectado y encargado del estudio para la definición del perfil profesional, debido a que aportan mayor conocimiento y repercuten en la realización de cambios en el proceso. 
Por otro lado, se integraron metodologías subyacentes eficientes que garantizaron el buen desarrollo y obtención de resultados de las fases del estudio. v) Para la identificación de los factores claves, se utilizó el diagrama de causa-efecto o Ishikawa, útil para la ordenación de ideas, por medio de sus relaciones de causalidad, el cual identificó las causas más probables que originaron el problema; vi) De la utilización del Diagrama de Pareto, a partir de la frecuencia que presentó cada causa, se determinaron los motivos principales que impedían el buen desarrollo de competencias en estudiantes de Ingeniería de Sistemas, las cuales fueron tenidas en cuenta al implementar las soluciones; vii) Al vincular la herramienta software SoftProsp, la participación de los actores de cada sector involucrado es anónima, activa y objetiva, lo cual permitió una definición apropiada y pertinente del perfil profesional.

\section{REFERENCIAS}

Agència per a la Qualitat del Sistema Universitari de Catalunya (AQU), Empleabilidad y Competencias de los Recién

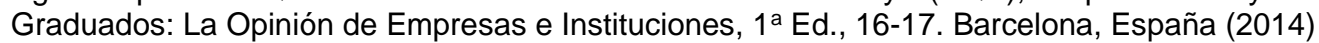

Álvarez, J. y Tello, C., Conocimientos, Actitudes e Implicancias Biopsicosociales y Éticas de la Ley 28704 en la Salud Sexual y Reproductiva de Mujeres Adolescentes Residentes del Distrito La Esperanza de Trujillo-Perú, ISNN: 2306-2002, Ciencia y Tecnología, 11(1), 99-112, 2015. https://goo.gl/uGVA2o. Acceso: 4 de enero (2018)

Ammons, D.N. y Roenigk, D.J., Benchmarking and Interorganizational Learning in Local Government, doi: 10.1093/jopart/muu014, Journal of Public Administration Research and Theory, 25(1), 309-335 (2014)

Anguita, J. C., Labrador, J. R., y J. D. Campos, La Encuesta como Técnica de Investigación. Elaboración de cuestionarios y tratamiento estadístico de los datos (I). Atención Primaria: 31(8), 527-538 (2003)

Anguita, J. C., Labrador, J. R., y J. D. Campos, La Encuesta como Técnica de Investigación. Elaboración de cuestionarios y tratamiento estadístico de los datos (II). Atención Primaria: 31(9), 592-600 (2003)

Assaf, G. y C. Pestana, A Global Benchmarking of the Hotel Industry, Tourism Economics, 19(4), $811-821$ (2013)

Assaf, G. y L. Dwyer, Benchmarking International Tourism Destinations, Tourism Economics, 19(6), 1233-1247 (2013)

Boned, P.C. y otros cuatro autores, Perfil Profesional de los Trabajadores de los Centros de Fitness en España, Rev. Internacional de Medicina de la Actividad Física y del Deporte, 15(58), 195-210 (2015)

Conde, C.R., Benchmarking de Mantenimiento en la Industria Química, Rev. Mantenimiento: ingeniería industrial y de edificios, (211), 20-26 (2008)

Córdoba, C.J., Desarrollo de Marca en Instituciones Educativas de Básica y Media: Una propuesta para el mejoramiento de la gestión educativa, Rev. e-ikon, 2(2), 17-35 (2014)

Dai, X. y T. Kuosmanen, Best-practice Benchmarking Using Clustering Methods: Application to energy regulation, doi: 10.1016/j.omega.2013.05.007, Omega, 42(1), 179-188 (2014)

Del Giorgio, F., Benchmarking Design: Multiplicación del Impacto de Asistencias Técnicas a MiPyMes en diseño y desarrollo de productos, Rev. Congreso Internacional de Diseño Industrial CIDI (2012)

Del Giorgio, F., Benchmarking en el Sector Público: Aportes y Propuestas de Implementación para la Provincia de Buenos Aires, $1^{a}$ Ed., 5-7. Industry Consulting Argentina, Buenos Aires, Argentina (2012)

Díaz-Bravo, L., y otros 3 autores, M. La Entrevista, Recurso Flexible y Dinámico, doi: 10.1016/S2007-5057(13)72706-6, Investigación en Educación Médica, 2(7), 162-167 (2013)

Edwards, R. A. y otros 5 autores, Using Benchmarking Techniques and the 2011 Maternity Practices Infant Nutrition and Care (mPINC) Survey to Improve Performance among Peer Groups across the United States, DOI: 10.1177/0890334413515948, Journal of Human Lactation, 30(1), 31-40 (2014)

Gómez, M.R., La Eficiencia del Sector en Michoacán desde la Óptica del Índice de Educación Municipal, 2000 y 2005, Rev. REICE: Revista Electrónica Iberoamericana sobre Calidad, Eficiencia y Cambio en Educación, 11(1), 20-39 (2013)

Gonzáles, G.O. y D.N. Patarroyo, Competencias Específicas Solicitadas al Recién Egresado de Ingeniería Industrial por el Sector Servicios en Bogotá, Rev. Ciencia e Ingeniería Neogranadina, 24(1), 163-179 (2014)

Gonzáles, K., A. Mortigo y N. Berdugo, La Configuración de Perfiles Profesionales en la Educación Superior y sus Implicaciones en el Currículo, Rev. Científica General José María Córdoba, 12(14), 165-182 (2014)

Hernández, R., C. Fernández y P. Baptista, Metodología de la Investigación, 4ª Ed., 102-210. McGraw-Hill, Iztapalapa, México (2006)

Jiménez, P.M. y B.T. González, El Cuestionario como Instrumento de Evaluación de Competencias Basado en la Evidencia Emocional de la Satisfacción, ISSN-e: 2341-4847. Aula de Encuentro, 2(17) 2015. https://goo.gl/gXZY4z. Acceso: 4 de Enero (2018)

Kuzmic, K., Benchmarking in University Development towards a Learning Organisation, International Journal of Contemporary Management, 14(2), 89-101 (2015)

Lapide, L., Benchmarking Best Practices, The Journal of Business, 6 (2005) 
Leppisaari, I., L. Vainio y J. Herrington, Virtual Benchmarking as Professional Development: Peer Learning in Authentic Learning Environments, Same Places, Different Spaces. Proceedings ascilite Auckland, 566 - 576 (2009)

MacLean, L., A. Richman y S. MacLean, Benchmarking Airports with Specific Safety Performance Measures, Transportation Research Part A: 92, 349-364 (2016)

Martelo, R.J., N. Villabona e I. Jiménez-Pitre, Guía Metodológica para Definir el Perfil Profesional de Programas Académicos mediante la Herramienta Ábaco de Régnier, en prensa (2016)

Martínez, T.N., La Referenciación Competitiva en la Evaluación de la Calidad de Enfermería, Rev. Cubana de enfermería, 32(2) (2016)

Ministerio de Educación Nacional, Articulación de la Educación con el Mundo Productivo, Revolución Educativa Colombia Aprende, Bogotá, Colombia (2014)

Morato, J., S. Sánchez-Cuadrado y M. Fernández-Bajón, Tendencias en el Perfil Tecnológico del Profesional de la Información, El profesional de la información, 25(2), 1699-2407 (2016)

Paliulis, N.K. y R. Labanauskis, Benchmarking as an Instrument for Improvement of Quality Management in Higher Education, Business, management and education, 13(1), 140-157 (2015)

Pérez, D., Perfil del Profesional Universitario Futuro Gerente PYME Fortaleza Sustentable para el Desarrollo Endógeno, Centro de Investigación de Ciencias Administrativas y Gerenciales CICAG, 7(2) (2010)

Santos, M.H., Una Herramienta Útil para Mejorar las Prácticas Educativas a Distancia, Rev. Mexicana de bachillerato a distancia, (14), (2015)

Soler-Adillon, J. y otros tres autores, Perfil del Profesional de la Comunicación Interactiva: Fundamentos, Actualidad y Perspectivas, Rev. El profesional de la información, 25(2), 196-208 (2016) 
